\title{
Impact of Multiphase Computed Tomography Angiography for Endovascular Treatment Decision- Making on Outcomes in Patients with Acute Ischemic Stroke
}

\author{
Johanna M. Ospel, ${ }^{\mathrm{a}, \mathrm{b}}$ Ondrej Volny, ${ }^{\mathrm{c}, \mathrm{d}}$ Wu Qiu, ${ }^{\mathrm{a}}$ Mohamed Najm, ${ }^{\mathrm{a}}$ Moiz Hafeez, ${ }^{\mathrm{a}}$ Sarah Abdalrahman, ${ }^{\mathrm{a}}$ \\ Enrico Fainardi, ${ }^{\mathrm{e}}$ Marta Rubiera, ${ }^{\mathrm{f}}$ Alexander Khaw, ${ }^{\mathrm{g}}$ Jai J. Shankar, ${ }^{\mathrm{h}}$ Michael D. Hill, ${ }^{\mathrm{a}, \mathrm{i}, \mathrm{j}, \mathrm{k}, \mathrm{l}}$ \\ Mohammed A. Almekhlafi, ${ }^{\mathrm{a}, \mathrm{i}, \mathrm{j}}$ Andrew M. Demchuk, ${ }^{\mathrm{a}, \mathrm{i}, \mathrm{j}}$ Mayank Goyal, ${ }^{\mathrm{a}, \mathrm{i}, \mathrm{j}}$ Bijoy K. Menon ${ }^{\mathrm{a}, \mathrm{i}, \mathrm{j}}$ \\ ${ }^{a}$ Department of Clinical Neurosciences, University of Calgary, Calgary, AB, Canada

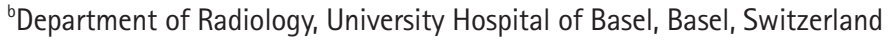 \\ ${ }^{c}$ Czech National Centre for Evidence-Based Healthcare and Knowledge Translation (Cochrane Czech Republic, Czech EBHC: JBI Centre of \\ Excellence, Masaryk University GRADE Centre), Institute of Biostatistics and Analyses, Faculty of Medicine, Masaryk University, Brno, Czech \\ Republic \\ ${ }^{\mathrm{d} D e p a r t m e n t}$ of Neurology, University Hospital Ostrava, Ostrava-Poruba, Czech Republic \\ 'Department of Experimental and Clinical Biomedical Sciences, University of Florence, Florence, Italy \\ fDepartment of Neurology, Hospital Vall d'Hebron, Barcelona, Spain \\ ${ }^{9}$ Department of Clinical Neurosciences, London Health Sciences Centre, University of Western Ontario, London, ON, Canada \\ ${ }^{\text {hDepartment }}$ of Radiology, University of Manitoba, Winnipeg, MB, Canada \\ 'Department of Radiology, University of Calgary, Calgary, $A B$, Canada \\ ${ }^{j}$ Hotchkiss Brain Institute, University of Calgary, Calgary, $A B$, Canada \\ ${ }^{k}$ Department of Community Health Sciences, University of Calgary, Calgary, $A B$, Canada \\ 'Department of Medicine, University of Calgary, Calgary, AB, Canada
}

Background and Purpose Various imaging paradigms are used for endovascular treatment (EVT) decision-making and outcome estimation in acute ischemic stroke (AIS). We aim to compare how these imaging paradigms perform for EVT patient selection and outcome estimation.

Methods Prospective multi-center cohort study of patients with AIS symptoms with multi-phase computed tomography angiography (mCTA) and computed tomography perfusion (CTP) baseline imaging. mCTA-based EVT-eligibility was defined as presence of large vessel occlusion (LVO) and moderate-to-good collaterals on mCTA. CTP-based eligibility was defined as presence of LVO, ischemic core (defined on relative cerebral blood flow, absolute cerebral blood flow, and cerebral blood volume maps) $<70 \mathrm{~mL}$, mismatch-ratio $>1.8$, absolute mismatch $>15 \mathrm{~mL}$. EVT-eligibility and adjusted rates of good outcome (modified Rankin Scale 0-2) based on these imaging paradigms were compared.

Results Of 289/464 patients with LVO, 263 (91\%) were EVT-eligible by mCTA-criteria versus 63 (22\%), 19 (7\%) and 103 (36\%) by rCBF, aCBF, and CBV-CTP-criteria. CTP and mCTA-criteria were discordant in 40\% to 53\%. Estimated outcomes were best in patients who met both mCTA and CTP eligibility-criteria and were treated with EVT (62\% to $87 \%$ good outcome). Patients eligible for EVT by mCTA-criteria and not by CTP-criteria receiving EVT achieved good outcome rates of 53\% to 57\%. Few patients met CTP-criteria and not mCTA-criteria for EVT.
Correspondence: Bijoy K. Menon Departments of Clinical Neurosciences and Radiology, University of Calgary Cumming School of Medicine, 1079 A, 29th St. NW, Calgary, AB T2N 2T9, Canada

Tel: +1-403-944 8107

Fax: +1-403-944 2611

E-mail:docbijoymenon@gmail.com https://orcid.org/0000-0002-3466-496X

Received: February 14, 2021

Revised: May 19, 2021

Accepted: July 16, 2021 
Conclusions Simpler imaging selection criteria that rely on little else than detection of the occluded blood vessel may be more sensitive and less specific, thus resulting in more patients being offered EVT and arguably benefiting from it.

Keywords Ischemic stroke; Ischemia; Cerebrovascular circulation

\section{Introduction}

The vanguard trials that established efficacy of endovascular treatment (EVT) in patients with acute ischemic stroke (AIS) used various imaging criteria for patient selection. These ranged from simple paradigms like non-contrast head computed tomography (NCCT) and single-phase computed tomography angiography (CTA) in the Multicenter Randomized Clinical trial of Endovascular treatment for Acute ischemic stroke in the Netherlands (MR CLEAN) ${ }^{1}$ and the EndoVascular Treatment With Stent-retriever and/or Thromboaspiration vs. Best Medical Therapy in Acute Ischemic Stroke (RESILIENT) trial (NCT02216643) to collateral imaging using multi-phase computed tomography angiography (mCTA) in the Endovascular Treatment for Small Core and Anterior Circulation Proximal Occlusion with Emphasis on Minimizing CT to Recanalization Times (ESCAPE) trial ${ }^{2}$ to multimodal magnetic resonance imaging (MRI) in the Trial and cost Effectiveness Evaluation of Intra-arterial Thrombectomy in Acute Ischemic Stroke (THRACE) trial. Computed tomography perfusion (CTP) was used exclusively in the Extending the Time for Thrombolysis in Emergency Neurological Deficits-Intra-Arterial (EXTEND IA) trial, ${ }^{3}$ a mixture of advanced imaging was used in the Solitaire ${ }^{\mathrm{TM}}$ With the Intention For Thrombectomy as PRIMary Endovascular Treatment (SWIFT PRIME) trial ${ }^{4}$ (some mCTA and some (TP) and the late window Clinical Mismatch in the Triage of Wake Up and Late Presenting Strokes Undergoing Neurointervention With Trevo (DAWN) ${ }^{5}$ and Endovascular Therapy Following Imaging Evaluation for Ischemic Stroke 3 (DEFUSE-3) trials had NCCT and CTP paradigms. Each approach has advantages and drawbacks but the lack of standardization of imaging paradigms globally results in misunderstanding about what types of patients are actually being enrolled into studies. This makes comparison of patient populations and treatment effects difficult, but it also constitutes an opportunity to compare different imaging paradigms and try to find an optimal imaging approach; one that provides just enough information for treatment decision-making and outcome prediction, without delaying or compromising treatment by either obtaining unnecessary information or excluding patients who may have benefited from treatment.

Of all the imaging paradigms in use in patients with acute stroke, NCCT with single phase CTA is the simplest and arguably the fastest, but reliability of assessment of the extent of ischemia is low, particularly among non-experts. Pial collateral status assessment has high specificity if the collaterals are good on a single phase CTA but poor collateral filling could be a false result due to delay in timing of the contrast bolus and consequent arterial filling. ${ }^{7}$ Both CTP and mCTA provide time-resolved images that try to address the issue of mistimed bolus contrast influencing assessment of contrast enhanced computed tomography (CT) in patients with acute stroke. ${ }^{8,9}$ In CTP, the brain is continuously scanned over 45 to 90 seconds, while in mCTA, three scan cycles are performed over 16 to 20 seconds. $^{8}$ Due to its higher temporal resolution, the information content in CTP images is higher when compared to mCTA, but this comes at the cost of lower spatial resolution, higher motion susceptibility and requirement for algorithm based image postprocessing.

Using a population of suspected AIS patients presenting within 12 hours of last known well, we compare CTP imaging for EVT decision-making to mCTA-based imaging to examine how much advanced imaging information is needed for patient selection and outcome prediction at a population level.

\section{Methods}

\section{Standard protocol approvals, registrations, and patient consents}

This study was approved by the local Institutional Review Board. The study protocol was registered on clinicaltrials.org (NCT02184936). The study was conducted according to the International Conference on Harmonization Guidelines for Good Clinical Practice and the Declaration of Helsinki. Patient consent was obtained prior to enrolment.

\section{Data availability}

Anonymized data will be made available by the corresponding author upon reasonable request.

\section{Study participants}

The Precise and Rapid Assessment of Collaterals Multi-phase CTA in the Triage of Patients With Acute Ischemic Stroke for IA Therapy (Prove-IT) study was a prospective multi-center cohort 
study that enrolled 595 patients who presented with AIS symptoms. Patients were included if they presented to the emergency department with symptoms consistent with AIS, were older than 18 years, and mCTA and CTP were both performed within 12 hours of symptom onset and before recanalization therapy. Exclusion criteria were intracranial hemorrhage at baseline NCCT, previous sizeable stroke in the ipsilateral hemisphere, modified Rankin Scale (mRS) $>2$ at baseline, estimated creatinine clearance $<60 \mathrm{~mL} / \mathrm{min}$, contrast material allergy, or other contraindications for iodinated contrast and estimated life expectancy $<1$ year. ${ }^{8}$ The enrollment period was July 2012 to October 2016.

\section{Imaging protocol}

\section{NCCT and $m$ CTA}

NCCT with $5 \mathrm{~mm}$ slice thickness was obtained, followed by a CTA with arch to vertex coverage (CTA Head and Neck; conventional single phase (TA). This first phase was followed by skull base to vertex coverage for the second (peak venous) and third (late venous) phase of a mCTA acquisition. Detailed mCTA acquisition parameters have been published previously. ${ }^{8}$ Axial images with $1 \mathrm{~mm}$ overlap and multiplanar axial, coronal and sagittal reconstructions with $3 \mathrm{~mm}$ thickness, $1 \mathrm{~mm}$ intervals, and $1 \mathrm{~mm}$ overlap for the first phase were obtained, along with axial minimum intensity projections for all three phases with $24 \mathrm{~mm}$ thickness and $4 \mathrm{~mm}$ intervals.

\section{CTP}

Forty-five milliliter of iodinated contrast agent were injected at a rate of $4.5 \mathrm{~mL} / \mathrm{sec}$ followed by a $40 \mathrm{~mL}$ saline bolus injected at a rate of $6 \mathrm{~mL} / \mathrm{sec}$. Image acquisition started 5 seconds after contrast injection and 24 passes over 66 seconds were performed with $5 \mathrm{~mm}$ section thickness and a cranio-caudal coverage of $8 \mathrm{~cm}$. CTP source data were centrally processed in a non-acute setting for each study using a delay-insensitive deconvolution software (CTP 4D; GE Healthcare, Waukesha, WI, USA). Time density curves were obtained and functional maps created, as described previously. Based on a review of existing literature, ischemic core volumes were then calculated using three different thresholds (relative cerebral blood flow [rCBF] $<30 \%$ from the EXTEND IA, SWIFT PRIME, and DEFUSE-3 trials $^{3,4,6}$ cerebral blood flow [CBF] $<7 \mathrm{~mL} / 100 \mathrm{~g} / \mathrm{min}$ based on a meta-analysis by Bandera et al., ${ }^{10}$ and cerebral blood volume [CBV] $<2 \mathrm{~mL} / 100 \mathrm{~g}$ based on a study by Wintermark et al. ${ }^{9}$ Penumbra volumes were calculated using the CTP $4 D$ equivalent of RAPID (iSchemaView, RAPID, Menlo Park, CA, USA) Tmax >6 seconds threshold. ${ }^{11}$

\section{Follow-up imaging}

Depending on the individual center's follow-up protocol, either diffusion-weighted MRI or NCCT images were obtained $24 \pm 6$ hours after initial presentation.

\section{Image analysis}

Images were assessed visually in three reading sessions (session 1, baseline NCCT only; session 2, baseline NCCT and MCTA; session 3, follow-up MRI/CT) with a 2 weeks interval between each of the sessions. Two raters (J.M.O., O.V.) read the images by consensus. Unclear findings were reviewed and interpreted by a senior neuroradiologist (M.G.). The readers had access to baseline clinical information (clinically affected hemisphere, National Institutes of Health Stroke Scale [NIHSS], time since symptom onset) during all reading sessions. They were blinded to other imaging and clinical outcomes. All CTP studies were interpreted with independent computerized methods. For a more detailed description of the image interpretation methodology see Supplementary material.

\section{Statistical methods}

We classified patients by EVT eligibility criteria defined by both: (1) NCCT+mCTA and (2) NCCT, single-phase CTA and CTP. Eligibility was defined by the presence of a large vessel occlusion (LVO) plus adequate collateral scores or appropriate perfusion criteria (Supplementary Table 1). The criteria for mCTA-based eligibility were derived from the ESCAPE and Safety and Efficacy of Nerinetide (NA-1) in Subjects Undergoing Endovascular Thrombectomy for Stroke (ESCAPE NA1) trials. ${ }^{2,12}$ Three criteria for CTP-based core definition were applied: ischemic core ( $\mathrm{rCBF}$ $<30 \%,{ }^{6} \mathrm{CBF}<7 \mathrm{~mL} / 100 \mathrm{~g} / \mathrm{min}^{10}$ and $\mathrm{CBV}<2 \mathrm{~mL} / 100 \mathrm{~g}^{9}$ ). The CTP 4D equivalent of RAPID iSchemaView Tmax $>6$ seconds was used as penumbra threshold. ${ }^{11}$ Cross tables and Venn diagrams were used to show modality-congruence between mCTA and CTP eligibility for EVT. Differences in proportions were assessed using the Fisher's exact test.

Co-primary outcomes were (1) independent outcome, defined as mRS score of 0-2 at 90 days and (2) major clinical improvement, defined as a 50\% relative reduction in the $\mathrm{NI}$ HSS score from baseline to 24-hour clinical follow-up. We chose major clinical improvement as a co-primary outcome a priori, ${ }_{1}^{8}$ based on prior published literature ${ }_{1}^{8}$ since it is more immediate, a direct reflection of treatment success and less likely to be confounded by differences in post-stroke rehabilitation and complications that occur during the 90-day followup period.

Patient baseline characteristics were reported using descriptive statistics. We constructed and compared a series of logis- 
tic regression models to assess the association between each imaging selection paradigm and the two pre-specified outcomes separately. A base model (model 1/"baseline model") was generated including pre-specified independent variables (age, baseline stroke severity as measured by the NIHSS, time from stroke symptom onset to baseline NCCT, baseline Alberta Stroke Program Early CT Score [ASPECTS; 11 point ordinal scale with lower values indicating greater degree of early ischemic changes] on NCCT, and occlusion location [anterior circulation LVO vs. not] and treatment type [EVT vs. not]). In other words, Model 1 included imaging information from NCCT (ASPECTS) and single-phase CTA only, which is the recommended imaging paradigm for patients with suspected AIS presenting in the early time window as per current guidelines. ${ }^{13,14}$ Each subsequent model incorporated imaging-based EVT eligibility criteria defined by each of the imaging threshold approaches described above as binary variables (eligible vs. ineligible). Model 2 included CTP criteria, with an ischemic core threshold of rCBF <30\%; Model 3 included CTP criteria, with an ischemic core threshold of $\mathrm{rCBF}<7 \mathrm{~mL} / 100 \mathrm{~g} / \mathrm{min}$; Model 4 included CTP criteria, with an ischemic core threshold of CBV $<2 \mathrm{~mL} / 100 \mathrm{~g} / \mathrm{min}$; Model 5 included mCTA EVT eligibility criteria. Models 6 through 8 were composite models that included each the above CTP eligibility criteria plus the MCTA EVT eligibility criteria. Akaike information criterion (AIC) and Bayesian information criterion (BIC) were then used to compare information loss across these five statistical models. AIC and BIC estimate the amount of information that is lost by a model while also considering the trade-off between the goodness of fit and simplicity of the model. Lower AIC and BIC scores indicate a better model. The $\mathrm{C}$ statistic was determined using receiver operating characteristics (ROC) for each of the above statistical models to describe the discriminative value (patient classification) of each model with higher C-statistics indicating a better model.

To understand the relative predictive value of each imaging criteria further, we constructed an 8-level nominal categorical variable for each of the models above (base model, three CTP approaches, mCTA approach, three CTP plus mCTA approaches). We used logistic regression, adjusted for patient age, baseline NIHSS, time from stroke symptom onset to baseline NCCT, baseline ASPECTS, occlusion location, and a dummy variable representing the eight possible combinations of $\mathrm{mCTA}$ eligibility (yes vs. no) versus CTP-eligibility (yes vs. no) conditional on treatment type (EVT performed vs. not) to assess the effect on outcome. This model helps generate probabilities for good outcomes for the following eight conditions namely (1) mCTA eligible, CTP eligible, EVT performed; (2) mCTA not eligible, CTP eligible, EVT performed; (3) mCTA not eligible, CTP eligible, EVT performed; (4) mCTA not eligible, CTP not eligible, EVT performed; (5) mCTA eligible, CTP eligible, EVT not performed; (6) mCTA not eligible, CTP eligible, EVT not performed; (7) mCTA not eligible, CTP eligible, EVT not performed; and (8) MCTA not eligible, CTP not eligible, EVT not performed. We deliberately chose to report adjusted rather than unadjusted outcomes since in a non-randomized setting, the latter are subject to substantial confounding by baseline prognostic variables. The effect size estimate using this approach is then relative to the base model and allows direct comparison of each imaging modality and treatment combination.

Finally, the net reclassification index (NRI) and integrated discrimination improvement (IDI) were calculated to compare imaging paradigms in predicting outcome to provide an estimate of the relative incremental benefit of each imaging approach. All analyses were performed in Stata version 15.1 (StataCorp., College Station, TX, USA). Two-sided $P$-values $<0.05$ were considered statistically significant. Results were visualized using Microsoft Excel version 16.18 (Microsoft, Redmond, WA, USA).

\section{Results}

\section{Study participants and outcomes}

Of the 595 patients enrolled in the study, complete clinical and imaging information were available in 464 patients, who were included in this analysis (Supplementary Figure 1). Patient baseline characteristics and treatment details are shown in Table 1. A detailed overview of the occlusion sites on baseline CTA is provided in Supplementary Table 2. Overall, 220 (47.4\%) patients were treated with EVT, either as single treatment or in combination with intravenous alteplase. Clinical outcome data are shown in Supplementary Table 3.

\section{Comparison of eligibility for EVT based on mCTA} vs. CTP criteria

Venn diagrams illustrating mCTA and CTP eligibility proportions when compared to the sample with LVOs and the entire sample are shown in Figure 1. Of 289/464 patients with LVO on CTA, $263(91 \%)$ were eligible for EVT by mCTA eligibility criteria versus $63(22 \%), 19(7 \%)$, and $103(36 \%)$ by rCBF, absolute cerebral blood flow (aCBF) and CBV CTP criteria. CTP and mCTA criteria were discordant in 44\% $(204 / 464, \mathrm{rCBF}<30 \%)$ (Figure 1A), 53.4\% (248/464, aCBF $<7 \mathrm{~mL} / 100 \mathrm{~g} / \mathrm{min}$ ) (Figure 1B), and $39.7 \%(184 / 464, C B V<2 \mathrm{~mL} / 100 \mathrm{~g} / \mathrm{min}$ ) (Figure $1 \mathrm{C}$ ), respectively. Most discordant patients were classified as EVT-eligible based on mCTA but not on CTP, regardless of the CTP threshold 
Table 1. Patient baseline characteristics and treatment details of the study sample $(n=464)$

\begin{tabular}{|c|c|}
\hline Characteristic & Value \\
\hline \multicolumn{2}{|l|}{ Clinical baseline } \\
\hline Age $(y r)$ & $73(62.5-80)$ \\
\hline Female sex & $219(47.2)$ \\
\hline Body mass index $\left(\mathrm{kg} / \mathrm{m}^{2}\right)(\mathrm{n}=324)$ & $27.9 \pm 11.9$ \\
\hline \multicolumn{2}{|l|}{ Medical history } \\
\hline Prior stroke or TIA & $88(19.0)$ \\
\hline Hypertension $(n=463)$ & $307(66.3)$ \\
\hline Diabetes mellitus $(n=460)$ & $70(15.2)$ \\
\hline Dyslipidemia $(n=462)$ & $182(39.4)$ \\
\hline Atrial fibrillation $(n=460)$ & $146(31.7)$ \\
\hline Chronic heart failure $(n=463)$ & $40(8.6)$ \\
\hline Valvular disease $(\mathrm{n}=463)$ & $28(6.1)$ \\
\hline Coronary artery disease $(n=463)$ & $83(17.9)$ \\
\hline Peripheral vascular disease $(n=461)$ & $27(5.9)$ \\
\hline Anticoagulant treatment $(n=458)$ & $64(14.0)$ \\
\hline Systolic blood pressure at admission $(\mathrm{mm} \mathrm{Hg})(\mathrm{n}=459)$ & $153.2 \pm 27.4$ \\
\hline Heart rate at admission (beats per minute) $(n=445)$ & $76(66-89)$ \\
\hline Glucose level at admission (mmol/L) $(\mathrm{n}=459)$ & $8.1 \pm 23.2$ \\
\hline NIHSS at admission & $13(6-20)$ \\
\hline Time from last known well to CT, median (range) (min) & $125(0-1,428)$ \\
\hline \multicolumn{2}{|l|}{ Imaging baseline } \\
\hline ASPECTS on NCCT ( $n=463)$ & $10(8-10)$ \\
\hline Hemorrhagic stroke at presentation $(n=463)$ & $1(0.2)$ \\
\hline Subacute infarct on NCCT at presentation $(n=463)$ & $12(2.6)$ \\
\hline \multicolumn{2}{|l|}{ Location of occlusion on multiphase CTA } \\
\hline Proximal anterior circulation (ICA, M1, and proximal M2) & $289(62.3)$ \\
\hline Distal anterior circulation (distal M2 and further distal) & $103(22.2)$ \\
\hline Posterior circulation & $23(5.0)$ \\
\hline No occlusion seen & $49(10.6)$ \\
\hline Secondary occlusion & $43(9.3)$ \\
\hline Multiple clots in the affected territory $(n=463)$ & $195(42.1)$ \\
\hline \multicolumn{2}{|l|}{ Type of treatment } \\
\hline Mechanical thrombectomy & $80(17.2)$ \\
\hline Intravenous alteplase & $132(28.5)$ \\
\hline Intravenous tenecteplase & $11(2.4)$ \\
\hline Mechanical thrombectomy and intravenous alteplase & $140(30.2)$ \\
\hline Supportive medical treatment only & $101(21.8)$ \\
\hline
\end{tabular}

Values are presented as median (interquartile range), number (\%), or mean \pm standard deviation.

TIA, transient ischemic attack; NIHSS, National Institutes of Health Stroke Scale; CT, computed tomography; ASPECTS, Alberta Stroke Program Early CT Score; NCCT, non-contrast head computed tomography; CTA, computed tomography angiography; ICA, internal carotid artery.

applied (rCBF, 99.0\% [202/204]; aCBF, 99.2\% [246/248]; CBV, 93.5\% [172/184]) (Figure 1). Results were very similar when only patients presenting beyond 6 hours $(n=69)$ were included (Supplementary Figure 2).
Association between imaging selection paradigms for EVT and clinical outcomes

Modelling results with the two co-primary clinical outcomes as dependent variables are shown in Tables 2 and 3. When assess- 
Grey circles: anterior circulation LVO

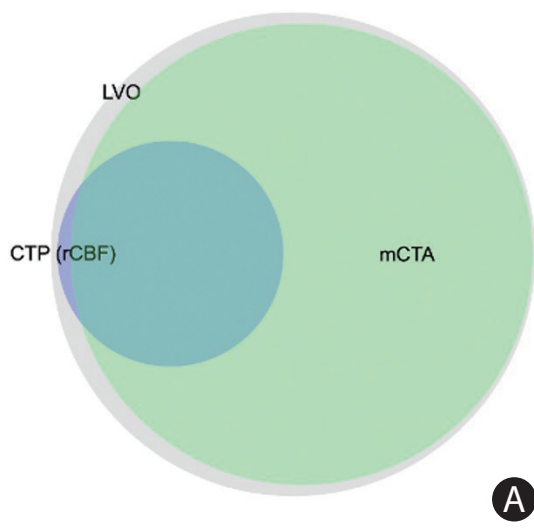

Green circles: eligible according to $\mathrm{mCTA}$

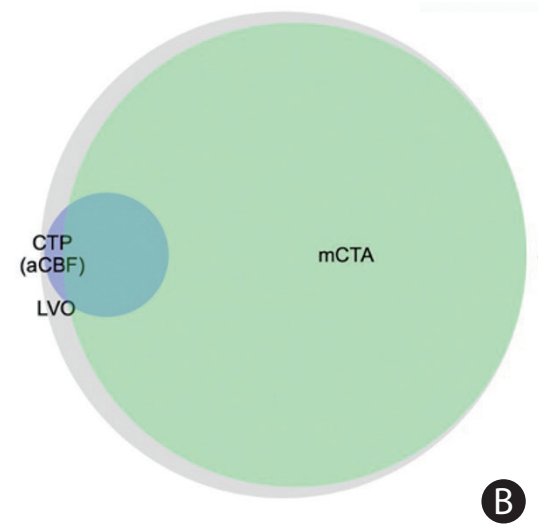

Blue circles: eligible according to CTP

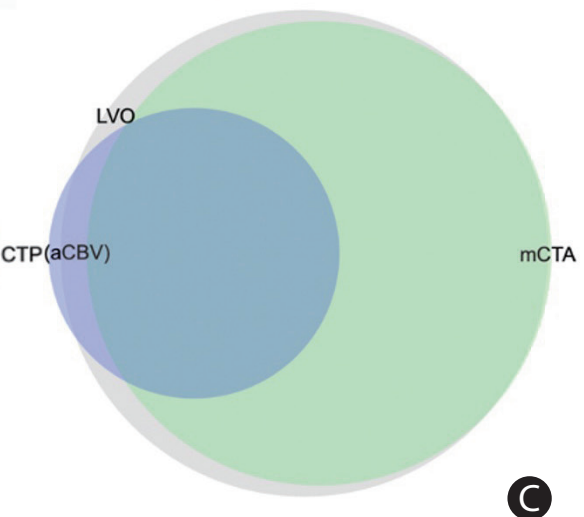

Figure 1. Proportional Venn diagrams illustrating the number of patients with anterior circulation large vessel occlusion (LVO), i.e., target for endovascular treatment (EVT; grey circles), and EVT eligible patients based on computed tomography perfusion (CTP) criteria (blue circles) and multi-phase computed tomography angiography (mCTA) criteria (green circles). (A) Illustrates CTP based EVT-eligibility for relative cerebral blood flow (rCBF) $<30 \%$ as core threshold, (B) illustrates CTP based EVT-eligibility for absolute cerebral blood flow (aCBF) $<7 \mathrm{~mL} / 100 \mathrm{~g} / \mathrm{min}$ as core threshold, and (C) illustrates CTP based EVT-eligibility for absolute cerebral blood volume (aCBV) $<2 \mathrm{~mL} / 100 \mathrm{~g}$ as core threshold.

ing 90-day mRS 0-2 as outcome, information loss was lowest with the CTP (aCBF) model (AIC, 486.60; BIC, 515.56) with the mCTA model being next lowest (AIC, 491.00; BIC, 519.96). Information loss with the other CTP based models was mostly higher and the $\mathrm{C}$ statistic lower than the rCBF and the mCTA based models (although the 95\% confidence intervals [Cls] for the $\mathrm{C}$ statistics overlap). When assessing major clinical improvement at 24 hours as outcome, information loss was lowest with the CTP (CBV) model (AIC, 610.44; BIC, 639.41) with the mCTA model being next lowest (AIC, 610.52; BIC, 639.48) while the $95 \% \mathrm{Cl}$ of the $\mathrm{C}$ statistics for all models overlapped. Results were similar after excluding posterior circulation strokes (data not shown) and in subjects with only anterior circulation LVO (Supplementary Table 4).

Estimated probabilities of pre-defined clinical outcomes (along with interquartile range), adjusted for pre-specified baseline variables and CTP versus mCTA eligibility criteria conditional on receiving EVT treatment, are shown in Figure 2. On average, in patients who received EVT, estimated outcomes were best in patients who met both MCTA and CTP eligibility criteria for EVT (62\% to 87\% good outcome rate and 57\% to $63 \%$ rate of major clinical improvement depending on the CTP threshold used). Patients eligible for EVT by mCTA criteria and not by CTP criteria who received EVT achieved adjusted good outcome rates of 53\% to $57 \%$ and major clinical improvement rates of $55 \%$ to $58 \%$. Very few patients met CTP eligibility criteria and not $\mathrm{mCTA}$ eligibility criteria for $\mathrm{EVT}(n=2,2$ and 12 for the $\mathrm{rCBF}_{\text {, }} \mathrm{CBBF}$, and $\mathrm{CBV}$ based thresholds, respectively). Patients who did not meet either mCTA or CTP eligibility criteria and received EVT achieved adjusted good outcome rates of
$51 \%$ to $62 \%$ and major clinical improvement rates of $42 \%$ to $49 \%$. Patients who met CTP or mCTA eligibility criteria but were not offered EVT were mainly patients with mild symptoms (NIHSS $\leq 7$ ), pre-stroke disability (baseline $m R S \geq 2$ ), long onset to imaging times ( $>6$ hours), extensive ischemic changes on baseline imaging (ASPECTS 6 or 7) and patients above 80 years of age (data not shown). Almost all patients ( $n=150 / 156,96 \%)$ who did not meet mCTA or CTP eligibility criteria and were not offered EVT did not have a LVO in the anterior circulation.

\section{Net reclassification analysis}

When comparing a statistical model that included baseline clinical information, NCCT ASPECTS, LVO presence, and mCTA based EVT eligibility criteria to another model that included the CTP based EVT eligibility criteria in addition, the NRI and IDI for major clinical improvement were small and not statistically significant (Table 4). The NRI was negative when $\mathrm{rCBF}<30 \%$ and $\mathrm{CBV}<2 \mathrm{~mL} / 100 \mathrm{~g}$ were used as ischemic core threshold, implying a worsening rather than an improvement in reclassification. Results for mRS 0-2 at 90 days were similar, except when $\mathrm{aCBF}<7 \mathrm{~mL} / 100 \mathrm{~g} / \mathrm{min}$ was used; here, the IDI was small but significant.

\section{Discussion}

This study finds that nine of every 10 patients with LVO presenting within 12 hours of symptom onset are considered eligible for EVT by mCTA criteria when compared to two of every 10 subjects considered eligible for EVT by rCBF based CTP criteria, four of every 10 subjects by CBV CTP criteria and one of 
Table 2. Statistical models* comparing the various imaging based eligibility criteria in estimating major clinical improvement, defined as 24-hour NIHSS drop $>50 \%$ compared to baseline $(n=464)$

\begin{tabular}{|c|c|c|c|}
\hline Variable & AIC & $\mathrm{BIC}$ & C statistic $(95 \% \mathrm{Cl})$ \\
\hline \multicolumn{4}{|l|}{ Statistical models* } \\
\hline Model 1. Baseline model (simplest) ${ }^{+}$ & 611.57 & 640.54 & $0.664(0.614-0.713)$ \\
\hline Model 2. Baseline model+CTP based eligibility for EVT (rCBF) & 611.36 & 640.32 & $0.670(0.621-0.719)$ \\
\hline Model 3. Baseline model+CTP based eligibility for EVT (aCBF) & 612.57 & 641.54 & $0.665(0.616-0.714)$ \\
\hline Model 4. Baseline model+CTP based eligibility for EVT (CBV) & 610.44 & 639.41 & $0.671(0.622-0.720)$ \\
\hline Model 5. Baseline model+mCTA based eligibility for EVT & 610.52 & 639.48 & $0.665(0.616-0.714)$ \\
\hline \multicolumn{4}{|l|}{ Baseline model ${ }^{+}+m C T A$ criteria vs. additional CTP criteria } \\
\hline Model 6. Model 5+CTP based EVT eligibility (rCBF) & 609.51 & 642.61 & $0.670(0.621-0.719)$ \\
\hline Model 7. Model 5+CTP based EVT eligibility (aCBF) & 612.49 & 645.60 & $0.664(0.615-0.713)$ \\
\hline Model 8. Model 5+CTP based EVT eligibility (CBV) & 611.20 & 644.30 & 0.669 (0.621-0.718) \\
\hline
\end{tabular}

NIHSS, National Institutes of Health Stroke Scale; AIC, Akaike information criterion; BIC, Bayesian information criterion; Cl, confidence interval; CTP, computed tomography perfusion; EVT, endovascular treatment; rCBF, relative cerebral blood flow; aCBF, absolute cerebral blood flow; CBV, cerebral blood volume; mCTA, multi-phase computed tomography angiography.

${ }^{*} m$ CTA: EVT treatment decision (yes is indicated by presence of a large vessel occlusion and good or intermediate collaterals), CTP treatment decision (yes is indicated by an ischemic core volume [defined as $\mathrm{rCBF}<30 \%$ for model 2 , a CBF $<7 \mathrm{~mL} / 100 \mathrm{~g} / \mathrm{min}$ for model 3, CBV $<2 \mathrm{~mL} / 100 \mathrm{~g}$ for model 4 ]) $<70 \mathrm{~mL}$ and absolute mismatch $>15 \mathrm{~mL}$ and penumbra (defined as Tmax $>6$ seconds)/core mismatch ratio $>1.8$ (Endovascular Therapy Following Imaging Evaluation for Ischemic Stroke 3 [DEFUSE-3] criteria ${ }^{6}$; ${ }^{+}$Adjustment was performed for the following pre-specified baseline variables: age, baseline stroke severity as measured by the NIHSS, time from stroke symptom onset to baseline non-contrast head computed tomography (NCCT), baseline Alberta Stroke Program Early CT Score (ASPECTS) (11 point ordinal scale) on NCCT, occlusion location (anterior circulation large vessel occlusion vs. not) and treatment type (EVT vs. not). Since large vessel occlusion presence was part of the mCTA and CTP-based treatment decision rules, it was not incorporated as an independent variable in models 2-8.

Table 3. Statistical models* comparing the various imaging based eligibility criteria in estimating good outcome, defined as modified Rankin Scale 0-2 at 90 days $(n=464)$

\begin{tabular}{|c|c|c|c|}
\hline Variable & AIC & $\mathrm{BIC}$ & C statistic $(95 \% \mathrm{Cl})$ \\
\hline \multicolumn{4}{|l|}{ Statistical models* } \\
\hline Model 1. Baseline model (simplest) ${ }^{+}$ & 490.95 & 519.91 & $0.808(0.769-0.847)$ \\
\hline Model 2. Baseline model+CTP based EVT eligibility (rCBF) & 491.09 & 520.05 & $0.808(0.769-0.847)$ \\
\hline Model 3. Baseline model+CTP based EVT eligibility (aCBF) & 486.60 & 515.56 & $0.812(0.774-0.851)$ \\
\hline Model 4. Baseline model+CTP based EVT eligibility (CBV) & 491.02 & 519.98 & $0.808(0.769-0.847)$ \\
\hline Model 5. Baseline model+mCTA treatment decision & 490.99 & 519.96 & $0.808(0.769-0.847)$ \\
\hline \multicolumn{4}{|l|}{ Baseline model $^{+}+m C T A$ criteria vs. additional CTP criteria } \\
\hline Model 6. Model 5+CTP based EVT eligibility (rCBF) & 492.95 & 526.05 & $0.808(0.769-0.847)$ \\
\hline Model 7. Model 5+CTP based EVT eligibility (aCBF) & 488.55 & 521.66 & $0.813(0.774-0.851)$ \\
\hline Model 8. Model 5+CTP based EVT eligibility (CBV) & 492.94 & 526.05 & $0.808(0.769-0.847)$ \\
\hline
\end{tabular}

AIC, Akaike information criterion; BIC, Bayesian information criterion; Cl, confidence interval; CTP, computed tomography perfusion; EVT, endovascular treatment; rCBF, relative cerebral blood flow; aCBF, absolute cerebral blood flow; CBV, cerebral blood volume; mCTA, multi-phase computed tomography angiography. *mCTA: EVT treatment decision (yes is indicated by presence of a large vessel occlusion and good or intermediate collaterals), CTP treatment decision (yes is indicated by an ischemic core volume [defined as $\mathrm{rCBF}<30 \%$ for model 2 , a CBF $<7 \mathrm{~mL} / 100 \mathrm{~g} / \mathrm{min}$ for model $3, \mathrm{CBV}<2 \mathrm{~mL} / 100 \mathrm{~g}$ for model 4 ]) $<70 \mathrm{~mL}$ and absolute mismatch $>15 \mathrm{~mL}$ and penumbra (defined as Tmax $>6$ seconds)/core mismatch ratio $>1.8$ (DEFUSE-3 criteria ${ }^{6}$ ); ${ }^{+}$Adjustment was performed for the following pre-specified baseline variables: age, baseline stroke severity as measured by the National Institutes of Health Stroke Scale, time from stroke symptom onset to baseline non-contrast head computed tomography (NCCT), baseline Alberta Stroke Program Early CT Score (ASPECTS) (11 point ordinal scale) on NCCT, occlusion location (anterior circulation large vessel occlusion vs. not) and treatment type (EVT vs. not). Since large vessel occlusion presence was part of the mCTA and CTP-based treatment decision rules, it was not incorporated as an independent variable in models 2-8.

every 20 subjects by aCBF CTP criteria. Clinical outcomes are likely best when patients meet both $\mathrm{mCTA}$ and CTP eligibility criteria for EVT and are offered EVT (57\% to 63\% rate of major clinical improvement, $62 \%$ to $87 \%$ rate of good outcome, depending on the CTP threshold used). Clinical outcomes in pa- tients who meet mCTA eligibility criteria for EVT but not CTP eligibility criteria (55\% to $58 \%$ rate of major clinical improvement, $53 \%$ to $57 \%$ rate of good outcome) and in patients who do not meet either mCTA or CTP eligibility criteria (major clinical improvement rates of $42 \%$ to $49 \%$ and good outcome rates 


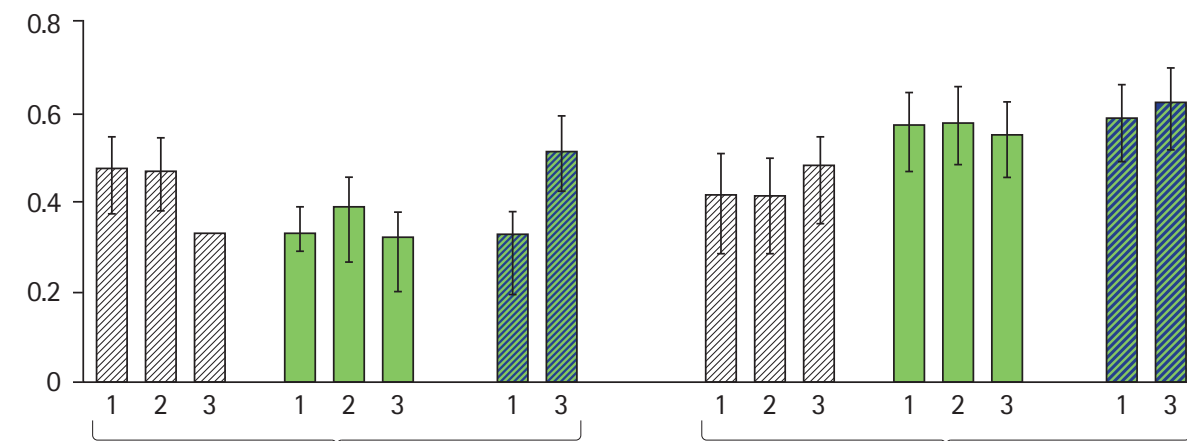

No EVT

EVT

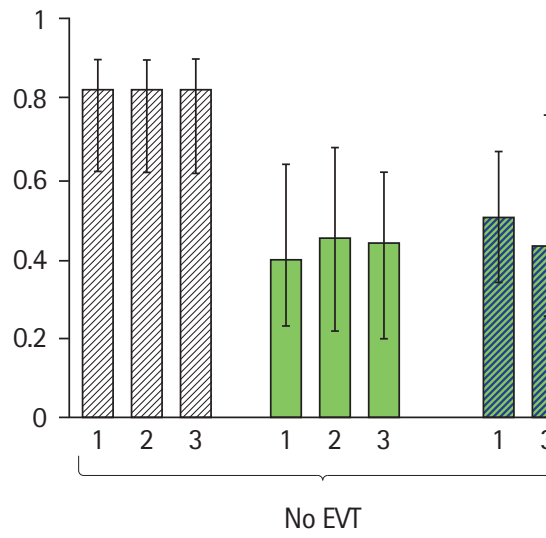

EVT

A

mCTA ineligble CTP ineligible

$\square$ mCTA eligible CTP ineligible

mCTA eligible CTP eligible

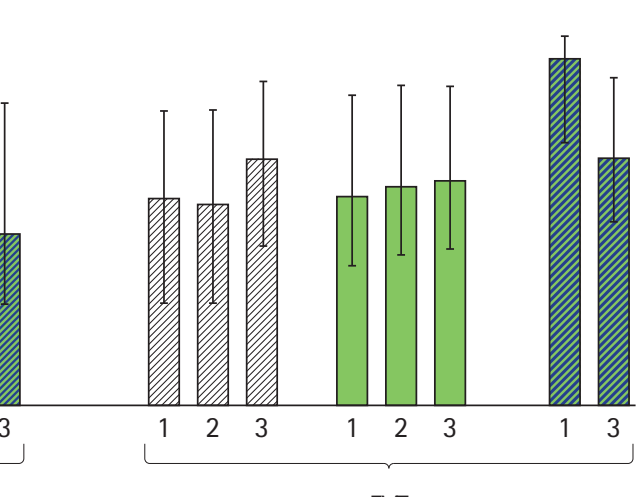

MCTA ineligble CTP ineligible

$\square$ mCTA eligible CTP ineligible

mCTA eligible CTP eligible

Figure 2. Median adjusted probability (model estimated) of major clinical improvement (A) and good outcome (B) conditional on computed tomography perfusion (CTP) vs. multi-phase computed tomography angiography (mCTA) based imaging selection criteria vs. both vs. neither and by treatment offered (endovascular treatment [EVT] vs. no EVT). Bars labelled with indicate probabilities for relative cerebral blood flow $<30 \%$ as core threshold; bars labelled with indicate probabilities for absolute cerebral blood flow $<7 \mathrm{~mL} / 100 \mathrm{~g} / \mathrm{min}$ as core threshold; and bars labelled with indicate probabilities for cerebral blood volume $<2 \mathrm{~mL} / 100 \mathrm{~g}$ as core threshold. Note that groups with less than 20 patients are not represented in this figure.

Table 4. Net reclassification index and integrated discrimination improvement for statistical models

\begin{tabular}{|c|c|c|c|c|}
\hline Additional parameter & NRI & $P$ & IDI & $P$ \\
\hline \multicolumn{5}{|l|}{ Dependent variable: major clinical improvement } \\
\hline CTP core volume (rCBF <30\%) & -0.011 & 0.677 & 0.005 & 0.123 \\
\hline CTP core volume (aCBF $<7 \mathrm{~mL} / 100 \mathrm{~g} / \mathrm{min})$ & 0.009 & 0.158 & $<0.001$ & 0.709 \\
\hline CTP core volume (CBV <2 mL/100 g) & -0.002 & 0.953 & 0.004 & 0.151 \\
\hline \multicolumn{5}{|l|}{ Dependent variable: good outcome } \\
\hline CTP core volume (rCBF <30\%) & 0.002 & 0.840 & $<0.001$ & 0.891 \\
\hline CTP core volume (aCBF $<7 \mathrm{~mL} / 100 \mathrm{~g} / \mathrm{min})$ & 0.018 & 0.260 & 0.012 & 0.014 \\
\hline CTP core volume (CBV <2 mL/100 g) & 0.007 & 0.448 & $<0.001$ & 0.767 \\
\hline
\end{tabular}

Net reclassification index and integrated discrimination improvement for a statistical model that included additional CTP eligibility criteria compared to a statistical model that included pre-specified baseline variables* and multi-phase computed tomography angiography based eligibility criteria (cut-off probability 50\%, dependent variables: major clinical improvement, defined as National Institutes of Health Stroke Scale [NIHSS] drop at 24 hours compared to baseline $>50 \%$, and good outcome, defined as modified Rankin Scale $0-2$ at 90 days). The analysis was conducted in the entire patient sample.

$\mathrm{NRI}$, net reclassification index; IDI, incremental discrimination improvement; CTP, computed tomography perfusion; rCBF, relative cerebral blood flow; aCBF, absolute cerebral blood flow; CBV, cerebral blood volume.

*Age, baseline stroke severity as measured by the NIHSS, time from stroke symptom onset to baseline non-contrast head computed tomography (NCCT), baseline Alberta Stroke Program Early CT Score (ASPECTS) (11-point ordinal scale) on NCCT and treatment type (endovascular treatment vs. not). 
of $51 \%$ to $62 \%$ ) are also good if they are offered EVT.

Acute stroke imaging serves two major purposes: treatment decision-making and prognostication. As opposed to prognostication, treatment decision making (patient selection for therapy) is a dichotomous process; either a decision is made in favor of, or against treatment. If imaging selection is to be considered valid, patients who are considered ineligible for treatment based on imaging should not benefit from that treatment. With the arguable exception of the MR CLEAN trial, all randomized controlled trials (including the late window DAWN and DEFUSE-3 trials) showing benefit of EVT in patients with LVOs used imaging selection criteria to exclude patients with LVOs from EVT. These imaging selection criteria range from the stringent e.g. CTP core and mismatch based criteria to the less stringent, e.g., mCTA or single phase CTA based criteria with NCCT ASPECTS criteria. Current guidelines suggest that additional imaging beyond NCCT and single-phase CTA is not required in the early time window. ${ }^{13,14}$ However, in current practice, CTP is often routinely obtained in all patients with suspected AIS, and there are several studies investigating the role of perfusion imaging in the early time window, ${ }^{15,16}$ including the ongoing randomized $A$ Randomized Controlled Trial to Optimize Patient's Selection for Endovascular Treatment in Acute Ischemic Stroke (SELECT-2) trial (NCT03876457). ${ }^{17}$ Our results show the potential impact of some of these various imaging selection criteria in excluding patients with LVOs presenting within 12 hours of last known well from EVT. More stringent imaging selection criteria result in better outcomes on average in patients selected for and offered EVT but at the cost of significantly less patients being offered EVT at the population level. Progressively less stringent imaging selection criteria result in more patients being offered EVT at the population level. Of note, even with the simplest imaging selection criteria that just seek to identify the existence of target LVO in patients, one in two patients achieve good clinical outcomes when offered EVT. In an earlier study, Kim et al. ${ }^{18}$ compared mCTA and CTP based triage in late window patients with confirmed LVO using the RAPID software, which defines ischemic core using an rCBF threshold of $<30 \%$ and a penumbra threshold of Tmax threshold of $>6$ seconds, and defined CTP based EVT eligibility using the DAWN and DEFUSE-3 criteria. They concluded that mCTA based EVT triage may be a good alternative to CTP based patient selection in the late time window. The present study evaluated additional CTP criteria and included a broader patient sample (patients with LVO, but also those with medium and small vessel occlusions, lacunar strokes, and stroke mimics), presenting both in the early and late time window. This study therefore provides additional, confirmatory evidence that im- aging strategies like NCCT/CTA or mCTA may be adequate for imaging based EVT triage, both in early and late time windows.

The fact that our results show approximately $50 \%$ to $62 \%$ good clinical outcome and $42 \%$ to $49 \%$ major clinical improvement rates in patients who are deemed ineligible for EVT by CTP or mCTA imaging criteria but were treated with EVT (when compared to 19\% good clinical outcome in the control arm of the MR CLEAN trial ${ }^{1}$ and $26.5 \%$ good clinical outcome in the control arm of the HERMES meta-analysis ${ }^{19}$ ) attests to the need for more careful consideration of the impact that current imaging selection criteria may have on health outcomes at a population level by excluding patients from robust treatments such as EVT. Other more recent analyses also support the idea that use of less stringent imaging selection criteria is likely to result in more patients receiving EVT and potentially benefiting from it, even in patients who present late. ${ }^{20}$ This raises the question as to what is then the use of more advanced imaging in acute stroke decision making? Our results show that, irrespective of the time window when patients present, statistical models for outcome determination that include imaging information from mCTA, CTP or a combination of these modalities have lower information loss and potentially better discrimination than models that do not include such information and rely only on ASPECTS and the presence of an LVO (the recommended triage strategy for early time window patients as per current guidelines), although the differences between these various models are minor. This suggests that information from more advanced imaging, i.e., imaging information that goes beyond mere detection of vessel occlusions, seems to have some additional utility for prognostication in patients with AIS.

Our results also show that very few patients $(\leq 3 \%)$ were eligible for EVT by CTP but not by mCTA. A patient level pooled analysis of data from the HERMES consortium of all recent EVT trials showed that patients with large ischemic cores on CTP may also benefit from EVT. ${ }^{21} \mathrm{~A}$ revision of current CTP criteria with a larger volume threshold for ischemic core and more liberal mismatch criteria may therefore agree more with the mCTA eligibility criteria presented here. Of note however, analysis of imaging criteria such as ASPECTS on NCCT and collateral status on CTA from the HERMES consortium did not show any treatment effect modification by imaging criteria, thus suggesting that patients who would otherwise be deemed ineligible for EVT by these imaging criteria may still benefit from EVT. ${ }^{22}$ Ongoing randomized controlled trials such as Efficacy and safety of ThrombEctomy iN Stroke with extended leSION and extended time window (TENSION), IN EXTREMIS, and Thrombectomy for Emergent Salvage of Large Anterior Circula- 
tion Ischemic Stroke (TESLA) are likely to shed further light on the appropriate use of imaging for patient selection for EVT.

A major strength of this study is that results are derived from a prospective multi-center cohort study with a large sample size and broad inclusion criteria. There are however some limitations to this study. First, patients were not randomly allocated to EVT versus medical management. We therefore do not know the "alternative" outcome had they been treated differently and could not directly compare the impact of CTP versus $\mathrm{mCTA}$ based EVT decision making on clinical outcome. Doing so would warrant a large diagnostic randomized controlled trial. ${ }^{23}$ Such a trial would be a pre-requisite for establishing the best imaging selection strategy for patients with LVOs presenting in the late time-window, but it would also be challenging to conduct, due the large required sample sizes and other methodological considerations. ${ }^{23}$ Second, assessment of CTP based EVT criteria in our study was fully automated but mCTA based EVT eligibility relied on visual scoring of collateral status. This may raise concern about between reader variability in assessing collateral status on mCTA. Although previous studies have shown that collateral assessment on mCTA by human raters may have good inter-rater reliability, ${ }^{8,18}$ automated collateral scoring systems that are increasingly available, will likely further mitigate concerns about reliability of such collateral assessment. ${ }^{24}$ Third, we used three different ischemic core definitions to represent a variety of currently used CTP parameters. Although these definitions are the most commonly used, the use of other ischemic core and penumbra thresholds may lead to different results. Fourth, only 69 subjects in this study presented beyond 6 hours of stroke symptom onset. Although we have shown previously that in these patients, the use of mCTA compares well with CTP in selecting patients for $\mathrm{EVT}_{1}{ }^{25}$ more data will be needed to compare these various available imaging paradigms in the late time window. The multicenter randomized clinical trial of endovascular treatment of acute ischemic stroke in The Netherlands for late arrivals (MR CLEAN LATE) (ISRCTN19922220) randomized controlled trial will provide further evidence in this regard.

\section{Conclusions}

In conclusion, this study suggests that simpler imaging selection criteria that rely on little else than detection of the occluded blood vessel may be more sensitive and less specific at a population level, thus resulting in more patients being offered EVT and arguably benefiting from it in patients with AIS and LVOs presenting within 12 hours of last known well. Advanced imaging however may have some additional value for prognostication in these patients.

\section{Supplementary materials}

Supplementary materials related to this article can be found online at https://doi.org/10.5853/jos.2021.00619.

\section{Disclosure}

Mayank Goyal: consultant (Medtronic, Stryker, Microvention, GE Healthcare, Mentice). Michael Hill: grants from Stryker, Medtronic, NoNO Inc., Boehringer Ingelheim. Bijoy Menon: patent (system/method for decision making/triaging in acute stroke). Johanna Ospel: research scholarships from the University of Basel Research Foundation, Julia Bangerter Rhyner Foundation and "Freiwillige Akademische Gesellschaft Basel." The remaining authors have nothing to disclose.

\section{Acknowledgments}

This study was supported by a grant from the Canadian Institute of Health Research. The authors are most grateful to all enrolling sites.

\section{References}

1. Berkhemer $O A$, Fransen PS, Beumer $D$, van den Berg $L A$, Lingsma $\mathrm{HF}$, Yoo $\mathrm{AJ}$, et al. A randomized trial of intraarterial treatment for acute ischemic stroke. N Engl J Med 2015;372: 11-20.

2. Goyal M, Demchuk AM, Menon BK, Eesa M, Rempel JL, Thornton J, et al. Randomized assessment of rapid endovascular treatment of ischemic stroke. N Engl J Med 2015;372: 1019-1030.

3. Campbell BC, Mitchell PJ, Kleinig TJ, Dewey HM, Churilov L, Yassi N, et al. Endovascular therapy for ischemic stroke with perfusion-imaging selection. N Engl J Med 2015;372:10091018.

4. Saver JL, Goyal M, Bonafe A, Diener HC, Levy El, Pereira VM, et al. Stent-retriever thrombectomy after intravenous t-PA vs. t-PA alone in stroke. N Engl J Med 2015;372:2285-2295.

5. Nogueira RG, Jadhav AP, Haussen DC, Bonafe A, Budzik RF, Bhuva $P$, et al. Thrombectomy 6 to 24 hours after stroke with a mismatch between deficit and infarct. N Engl J Med 2018; 378:11-21.

6. Albers GW, Marks MP, Kemp S, Christensen S, Tsai JP, OrtegaGutierrez S, et al. Thrombectomy for stroke at 6 to 16 hours with selection by perfusion imaging. N Eng/ J Med 2018;378: 
708-718.

7. Wang Z, Xie J, Tang TY, Zeng CH, Zhang Y, Zhao Z, et al. Collateral status at single-phase and multiphase CT angiography versus CT perfusion for outcome prediction in anterior circulation acute ischemic stroke. Radiology 2020;296:393-400.

8. Menon BK, d'Esterre CD, Qazi EM, Almekhlafi M, Hahn L, Demchuk AM, et al. Multiphase CT angiography: a new tool for the imaging triage of patients with acute ischemic stroke. Radiology 2015;275:510-520.

9. Wintermark M, Flanders $A E$, Velthuis $B$, Meuli $R$, van Leeuwen $M$, Goldsher $D$, et al. Perfusion-CT assessment of infarct core and penumbra: receiver operating characteristic curve analysis in 130 patients suspected of acute hemispheric stroke. Stroke 2006;37:979-985.

10. Bandera E, Botteri M, Minelli C, Sutton A, Abrams KR, Latronico N. Cerebral blood flow threshold of ischemic penumbra and infarct core in acute ischemic stroke: a systematic review. Stroke 2006;37:1334-1339.

11. d'Esterre CD, Boesen ME, Ahn SH, Pordeli P, Najm M, Minhas $P$, et al. Time-dependent computed tomographic perfusion thresholds for patients with acute ischemic stroke. Stroke 2015;46:3390-3397.

12. Hill MD, Goyal M, Menon BK, Nogueira RG, McTaggart RA, Demchuk AM, et al. Efficacy and safety of nerinetide for the treatment of acute ischaemic stroke (ESCAPE-NA1): a multicentre, double-blind, randomised controlled trial. Lancet 2020; 395:878-887.

13. Powers WJ, Rabinstein AA, Ackerson T, Adeoye OM, Bambakidis NC, Becker K, et al. Guidelines for the early management of patients with acute ischemic stroke: 2019 update to the 2018 guidelines for the early management of acute ischemic stroke: a guideline for healthcare professionals from the American Heart Association/American Stroke Association. Stroke 2019;50:e344-e418.

14. Turc G, Bhogal P, Fischer U, Khatri P, Lobotesis K, Mazighi M, et al. European Stroke Organisation (ESO): European Society for Minimally Invasive Neurological Therapy (ESMINT) guidelines on mechanical thrombectomy in acute ischemic stroke. J Neurointerv Surg 2019;11:535-538.

15. Olivot JM, Albucher JF, Guenego A, Thalamas C, Mlynash M, Rousseau $\mathrm{V}$, et al. Mismatch profile influences outcome after mechanical thrombectomy. Stroke 2021;52:232-240.

16. Sarraj $A$, Hassan $A E$, Savitz $S$, Sitton $C$, Grotta J, Chen $P$, et al.
Outcomes of endovascular thrombectomy vs medical management alone in patients with large ischemic cores: a secondary analysis of the optimizing patient's selection for endovascular treatment in acute ischemic stroke (SELECT) study. JAMA Neurol 2019;76:1147-1156.

17. Jadhav AP, Hacke W, Dippel DWJ, Simonsen CZ, Costalat V, Fiehler J, et al. Select wisely: the ethical challenge of defining large core with perfusion in the early time window. J Neurointerv Surg 2021;13:497-499.

18. Kim B, Jung C, Nam HS, Kim BM, Kim YD, Heo JH, et al. Comparison between perfusion- and collateral-based triage for endovascular thrombectomy in a late time window. Stroke 2019;50:3465-3470.

19. Goyal M, Menon BK, van Zwam WH, Dippel DW, Mitchell PJ, Demchuk AM, et al. Endovascular thrombectomy after largevessel ischaemic stroke: a meta-analysis of individual patient data from five randomised trials. Lancet 2016;387:17231731.

20. Lopez-Rivera V, Abdelkhaleq R, Yamal JM, Singh N, Savitz SI, Czap $A L$, et al. Impact of initial imaging protocol on likelihood of endovascular stroke therapy. Stroke 2020;51:30553063.

21. Menon BK, Hill MD, Davalos A, Roos YB, Campbell BC, Dippel $D W$, et al. Efficacy of endovascular thrombectomy in patients with M2 segment middle cerebral artery occlusions: metaanalysis of data from the HERMES Collaboration. J Neurointerv Surg 2019;11:1065-1069.

22. Román $L S$, Menon $B K$, Blasco J, Hernández-Pérez $M$, Dávalos $A$, Majoie $C B$, et al. Imaging features and safety and efficacy of endovascular stroke treatment: a meta-analysis of individual patient-level data. Lancet Neurol 2018;17:895-904.

23. Rodger $M$, Ramsay $T$, Fergusson D. Diagnostic randomized controlled trials: the final frontier. Trials 2012;13:137.

24. Grunwald IQ, Kulikovski J, Reith W, Gerry S, Namias R, Politi $M$, et al. Collateral automation for triage in stroke: evaluating automated scoring of collaterals in acute stroke on computed tomography scans. Cerebrovasc Dis 2019;47:217-222.

25. Almekhlafi MA, Kunz WG, McTaggart RA, Jayaraman MV, Najm $\mathrm{M}, \mathrm{Ahn} \mathrm{SH}$, et al. Imaging triage of patients with latewindow (6-24 hours) acute ischemic stroke: a comparative study using multiphase CT angiography versus CT perfusion. Am J Neuroradiol 2020;41:129-133. 


\section{Supplementary material}

\section{Imaging reading methods}

The readings described below were performed as consensus readings of two readers (one radiology resident and one neurology stroke fellow). Images were reviewed under appropriate conditions (dimmed light, multi monitor setup, windowing tools). Between the non-contrast head computed tomography (NCCT), the multi-phase computed tomography angiography (CTA) and the follow-up readings, reading was paused for 2 weeks respectively.

\section{NCCT reading}

$5 \mathrm{~mm}$ reconstructed axial unenhanced head computed tomography (CT) images were available with sagittal and coronal reformations. The readers had access to the side that was clinically affected, the National Institutes of Health Stroke Scale (NIHSS) score and the time of symptom onset and time of CT acquisition. The readers were blinded to the CTA, computed tomography perfusion (CTP), digital subtraction angiography (DSA), follow-up magnetic resonance (MR) or CT images and to clinical outcome data.

\section{Recorded items}

1) Alberta Stroke Program Early CT Score (ASPECTS): The ASPECTS was scored on NCCT images to assess the extent of early ischemic changes. Areas with early ischemic changes (relative hypodensity, loss of grey white matter differentiation, and effacement of sulci) were considered as affected and the regions affected were reported explicitly.

2) Affected side: The affected side (side of early ischemic changes and/or side of hyperdense or unsure hyperdense vessel sign) was reported. In case of early ischemic changes in the anterior circulation it was reported whether the right or left side was affected. In case of supratentorial and cerebellar posterior circulation changes, the affected side (right or left) was reported as well. In case of brainstem early ischemic changes, no side was reported.

3) Baseline hemorrhage: The presence of hemorrhage (defined as hyperdense foci with a typical morphology and blood equivalent Hounsfield unit [HU] values of 50-80) on the baseline NCCT was reported and in case of a (1) parenchymal hemorrhage, the side (right, left), location (frontal, parietal, temporal, occipital, limbic lobe, insula, deep grey matter, cerebellum, pons, mesencephalon, medulla); (2) subarachnoid hemorrhage, the side (right, left), location (frontal, parietal, temporal, occipital, limbic lobe, insula, infratentorial); (3) subdural hemorrhage, the side (right, left), location (frontal, parietal, temporal, occipital, infratentorial); (4) epidural hemorrhage, the side (right, left), location (frontal, parietal, temporal, occipital, infratentorial); and (5) intraventricular hemorrhage, the side (right, left, midline), affected ventricles/cisterns (right or left lateral ventricle, 3rd ventricle, aqueduct, 4th ventricle, prepontine/perimesencephalic or any other cistern) was reported.

4) Subacute infarcts: The presence of subacute infarcts (defined as sharply hypodense demarcated areas with a typical vascular distribution) was recorded. In case subacute infarcts were present, the side (right or left) and ASPECTS region in which the subacute infarct was present was recorded. In case of subacute infarcts in the posterior circulation, for supratentorial and cerebellar lesions, the side (right or left) and territory (occipital lobe, mesiotemporal lobe, deep grey matter, cerebellum) was reported. For brainstem lesions, no side was reported.

5) Hyperdense vessel sign: The presence of a hyperdense vessel sign was reported. It was noted whether a clear, unambiguous hyperdense vessel or no hyperdense vessel was seen.

Ambiguous cases were reported as unsure. In case of presence of a hyperdense or unsure hyperdense vessel sign, the vessel was noted as follows: (1) internal carotid artery (ICA; intracranial ICA); (2) middle cerebral artery (MCA) (hyperdensity which extends into the M1 and M2 portion); (3) M1 (hyperdensity only in the presumed localization of the M1 portion); (4) M2 (hyperdensity only in the presumed localization of the M2 portion); (5) B (hyperdensity only in the presumed localization of the basilar artery); (6) P1 (hyperdensity only in the presumed localization of the P1 portion); (7) P2 (hyperdensity only in the presumed localization of the P2 portion); and (8) V (hyperdensity only in the presumed localization of the vertebral artery).

\section{Multiphase CTA reading}

Five millimeter reconstructed axial unenhanced head CT images were available with sagittal and coronal reformations. Multiphase CTA images. Multiphase images (section thickness $0.625 \mathrm{~mm}$ ) were also available; an arch to vertex CTA constituted the first phase that was acquired during the peak arterial phase in the healthy brain parenchyma and was triggered by bolus monitoring. Coronal and sagittal reformations for the first phase were available. The second (peak venous) and third (late venous) phase were acquired after 11 and 19 seconds. Eighty milliliter of contrast were injected at a rate of $5 \mathrm{~mL} / \mathrm{sec}$ followed by a $50 \mathrm{~mL}$ saline bolus at a rate of $6 \mathrm{~mL} / \mathrm{sec}$. Axial images were reconstructed for all three phases with $1 \mathrm{~mm}$ overlap and multiplanar reconstructions for axial, coronal, and 
sagittal images of the circle of Willis were performed with 3-mm thickness at 1-mm intervals. Thick-section axial maximum intensity projections at 24-mm thickness and 4-mm intervals were also reconstructed and reviewed.

\section{Recorded items}

1) Affected side: The side (right or left) of the vessel occlusion was reported in case of anterior circulation occlusions and posterior cerebral artery (PCA) occlusions. In case of vertebral and basilar occlusions, no side provided. In case of no evident vessel occlusion, a side was reported if there were clear early or subacute ischemic changes on one side in the infra- or supratentorial parenchyma. In case of venous thrombosis, the side was provided for the sigmoid and transverse sinus, whereas no side was provided for the superior, inferior sagittal sinus and sinus rectus.

2) Primary occlusion: The primary occlusion was defined as the most proximal occlusion that subsequently affects the largest brain parenchyma volume. The most proximal end of the clot was decisive for the clot localization (e.g., a clot that extends from the ICA in the M1 and M2 was reported as an ICA occlusion). The vessels were noted as follows: (1) ICA: Intra- or extracranial ICA. The beginning was defined as the carotid bifurcation and the end as the ICA bifurcation; (2) M1: Begins distal to the ICA bifurcation and ends at the main bifurcation in the inferior and superior M2 branch/at the trifurcation in case of a trifurcation; (3) Proximal M2: M2 segment extending from the MCA main bifurcation up to $1 \mathrm{~cm}$ distal to the end of the M1 segment, easily accessible for endovascular treatment; (4) Distal M2: M2 segment more distal than $1 \mathrm{~cm}$ distal to the end of the $\mathrm{M} 1$ segment, ends at the circular sulcus of the insula; (5) M3: Begins at the circular sulcus of the insula and ends at the external surface of the Sylvian fissure; (6) M4: Begins distal to the M3 that reaches the external surface of the Sylvian fissure, superficially coursing branches at the lateral convexity; (7) A1: Starts at the ICA bifurcation, ends at the origin of the anterior communicating artery; (8) A2: Starts at the origin of the anterior communicating artery, ends at the genu of the corpus callosum; (9) A3: Starts at the genu of the corpus callosum, ends at the posterior turn of the artery, where it courses above the corpus callosum; (10) A4: Branches above the body of the corpus callosum; (11) P1: Origins at the terminal bifurcation of the basilar artery, ends at the origin of the posterior communicating artery; (12) P2: Starts at the origin of the posterior communicating artery and courses around themesencephalon ends when the vessel enters the quadrigeminal cistern; (13) P3: Starts when the vessel enters the quadrigeminal cistern, courses in the quadrigeminal cistern; (14) P4: Starts when the branches enter the cortex, cortical branches; (15) Basilar artery: Starts at the junction of the vertebral arteries and ends at the basilar bifurcation; and (16) Vertebral artery: Start at its origin from the subclavian artery (or aortic arch on the leftside) and ends at the vertebrobasilar junction.

3) Clot burden score: The clot burden score was reported in patients with anterior circulation vessel occlusion. In case of no evident occlusion, the clot burden score was not reported. In case posterior circulation and distal anterior circulation ( $M 3$ and $M 4, A 2, A 3, A 4)$ occlusions, the clot burden score was reported as 10 .

4) Secondary occlusion: In case of a second, major occlusion with clear flow affection in the downstream territory attributable to this occlusion, this was noted as a secondary occlusion. The occlusion localization was noted as for primary occlusions.

5) Side of secondary occlusion: The occlusion side was noted as for primary occlusions (right or left for anterior circulation and PCA occlusions, no side was reported for vertebral and basilar occlusions).

6) Pial arterial filling (collateral grading): The delay and extent of collateral filling for the primary occlusion was graded on axial maximum intensity projections of the three multi-phase computed tomography angiography phases. Grading was as follows: (Grade 0) Poor collaterals: no or only few vessels visible in any phase within the occluded vascular territory compared to the asymptomatic contralateral hemisphere; (Grade 1) Intermediate collaterals: delay of two phases in filling in of peripheral vessels with or without decreased prominence and extent or a one-phase delay and some ischemic regions with no only few or no vessels compared to the asymptomatic contralateral hemisphere; (Grade 2) Good collaterals: no delay or 1 phase delay in filling of peripheral vessels with identical or increased prominence of vessels compared to the asymptomatic contralateral hemisphere.

In case of posterior circulation occlusions, this item was not reported.

\section{Follow-up reading}

Either magnetic resonance imaging (MRI) (susceptibilityweighted or gradient echo images, diffusion weighted images and apparent diffusion coefficient images and fluid attenuated inversion recovery images) or unenhancedhead CT images that were obtained $24( \pm 6)$ hours after initial imaging were reviewed. The readers had access to the side that was clinically affected, but were blinded to baseline NCCT; CTA and CTP im- 
ages and DSA images.

Recorded items

1) Imaging modality: It was noted whether the follow-up imaging was either a CT or an MR. In case both existed, the MR was chosen for evaluation.

2) Final ASPECTS: The follow-up ASPECTS was scored. A region was called affected when at least $30 \%$ of it was affected and the affected regions were reported explicitly. (1) CT: A region was called affected when there was clear, sharp hypodense demarcation of at least 30\% of the parenchyma, with or without hemorrhage. Regions with changes indicative of older infarcts like encephalomalacia or old calcified infarcts were not considered affected. (2) MRI: A region was called affected if there was DWI-hyperintense signal in at least $30 \%$ of the parenchyma. In case of bilateral lesions, the side with the larger affected territory was reported. In case of ischemic changes in the posterior circulation and in the anterior cerebral artery (ACA) territory, the ASPECTS was recorded as 10.

3) Side: The side of ischemic changes were reported for anterior circulation and PCA territory infarcts. For brainstem lesions, no side was reported. In case of bilateral ischemic changes, the side with the larger affected territory was reported. If the extent of ischemic changes was equal on both sides, this was reported as both sides. In case no new or subacute ischemic lesions could be identified, this was reported as no changes.

4) Ischemic changes in the ACA territories: It was reported whether ischemic changes in theACA territory were present (either isolated or in addition to ischemic changes in other territories). In case they were present, the side (left, right, or both) was reported.

5) Ischemic changes in the vertebrobasilar territory: It was reported whether ischemic changes in the vertebrobasilar territory were present (either isolated or in addition to ischemic changes in other territories). In case they were present, for PCA territory lesions, the side (left, right, orboth) was reported. For brainstem lesions, no side was reported.

6) Hemorrhagic transformation: For $C T$, the presence and degree of hemorrhagic transformation according to the The European Cooperative Acute Stroke Study (ECASS) criteria and an extension of the classification was used for reviews of follow-up MRIs. The scans were assigned to each of the following categories: no hemorrhagic transformation, hemorrhagic infarction type 1 , hemorrhagic infarction type 2 , parenchymal hemorrhage type 1, parenchymal hemorrhage type 2. In case both MR and CT follow-up imaging was available, MRI was used. In order to distinguish contrast staining post thrombectomy and hemorrhage on $\mathrm{CT}$, density measurements of the affected area were taken and areas with $\mathrm{HU}$ values $50-80$ considered as hemorrhage, whereas much higher values (above $120 \mathrm{HU}$ ) were considered contrast staining.

7) Remote parenchymal hemorrhage: The presence of parenchymal hemorrhage in areas remote from the ischemic changes was reported. In case they were present, the side (right, left) and location (frontal, parietal, temporal, occipital, limbic lobe, insula, deep grey matter, cerebellum, pons, mesencephalon, medulla) was reported.

8) Other hemorrhage: The presence and type of extraaxial hemorrhage (subarachnoid, subdural, epidural, intraventricular) was reported. In case an extraaxial hemorrhage was present, the side (right, left) and location (frontal, parietal, temporal, occipital, limbic lobe, insula, infratentorial) was reported.

\section{Additional statistical methods (reclassification analysis)}

The net reclassification index (NRI) and integrated discrimination improvement (IDI) were calculated to compare imaging paradigms in predicting outcome to provide an estimate of the relative incremental benefit of each imaging approach. The NRI assigns a positive value for a model that correctly reclassifies a patient, while those who are incorrectly reclassified are assigned a negative value. Thus, a positive NRI indicates reclassification improvement compared to the base model, while a negative value indicates worsening. The magnitude of the NRI corresponds to the extent of improvement/worsening. The IDI represents the difference in discrimination slopes: it expresses how much a new model increases the assigned risk in patients with events and decreases the assigned risk in patients without eventscompared to the base model. Reclassification analysis was attempted for both outcomes.

Two-sided $P$-values < 0.05 were considered statistically significant. 
Supplementary Table 1. EVT-eligibility criteria for different imaging modalities

\begin{tabular}{ll}
\hline Imaging modality & EVT eligibility criteria (all have to apply) \\
\hline $\mathrm{mCTA}$ & Presence of a large vessel occlusion in the anterior circulation (internal carotid artery, M1 or proximal \\
& $\mathrm{M} 2$ middle cerebral artery occlusion) \\
& Good or intermediate collaterals (defined as $>25 \%$ filling of the middle cerebral artery territory) \\
Single phase CTA+CTP & Presence of a large vessel occlusion in the anterior circulation (internal carotid artery, M1 or proximal \\
Ischemic core thresholds: & M2 middle cerebral artery occlusion) \\
$\mathrm{rCBF}<30 \%$ & Ischemic core volume $<70 \mathrm{~mL}$ \\
$\mathrm{aCBF}<7 \mathrm{~mL} / 100 \mathrm{~g} / \mathrm{min}$ & Mismatch ratio $>1.8$ \\
$\mathrm{CBV}<2 \mathrm{~mL} / 100 \mathrm{~g}$ & Absolute mismatch $>15 \mathrm{~mL}$ \\
Penumbra threshold: Tmax $>6$ seconds &
\end{tabular}

EVT, endovascular treatment; mCTA, multi-phase computed tomography angiography; CTA, computed tomography angiography; CTP, computed tomography perfusion; rCBF, relativecerebral blood flow; aCBF, absolute cerebral blood flow; $\mathrm{CBV}$, cerebral blood volume.

Supplementary Table 2. Detailed location of intracranial vessel occlusion sites $(n=464)$

\begin{tabular}{lc}
\hline Variable & No. $(\%)$ \\
\hline Anterior circulation & $58(12.5)$ \\
Internal carotid artery & $186(40.1)$ \\
M1 segment & $45(9.7)$ \\
Proximal M2 segment & $37(8.0)$ \\
Distal M2 segment & $38(8.2)$ \\
M3 segment & $19(4.1)$ \\
M4 segment & $4(0.9)$ \\
A2 segment & $2(0.4)$ \\
A3 segment & $3(0.7)$ \\
A4 segment & \\
Posterior circulation & $4(0.9)$ \\
Vertebral artery & $4(0.9)$ \\
Basilar artery & $2(0.4)$ \\
P1 segment & $12(2.6)$ \\
P2 segment & $1(0.2)$ \\
Superior cerebellar artery & $49(10.6)$ \\
No occlusion seen & \\
\hline
\end{tabular}


Supplementary Table 3. Clinical and imaging outcomes of the study sample $(n=464)$

\begin{tabular}{lc}
\hline Clinical and imaging outcome data & Value \\
\hline$m R S$ at 90 days & $2(1-4)$ \\
mRS $0-2$ at 90 days & $284(61.2)$ \\
NIHSS at 24 hours & $5(2-13)$ \\
Major clinical improvement & $219(47.2)$ \\
Recanalization rate & $68 / 331(20.5)$ \\
rAOL $2 b / 3$ ( $n=331)$ & $64 / 224(28.6)$ \\
eTICl 2c/3 $(n=224)$ & $8(7-10)$ \\
Final ASPECTS ( $n=455)$ & $32.8(62.6)$ \\
Final infarct volume $(\mathrm{mL})(\mathrm{n}=432)$ & $36(7.9)$ \\
Parenchymal hemorrhage $(n=455)$ & $3(0.7)$ \\
Remote parenchymal hemorrhage $(n=455)$ &
\end{tabular}

Values are presented as median (interquartile range) or number (\%).

mRS, modified Rankin Scale; NIHSS, National Institutes of Health Stroke Scale; rAOL, revised Arterial Occlusive Lesion; eTICl, expanded Treatment in Cerebral Infarction; ASPECTS, Alberta Stroke Program Early CT Score.

Supplementary Table 4. Predicting major clinical improvement with imaging

\begin{tabular}{|c|c|c|c|}
\hline Statistical models* & AIC & $\mathrm{BIC}$ & C statistic $(95 \% \mathrm{Cl})$ \\
\hline \multicolumn{4}{|l|}{ Major clinical improvement } \\
\hline Model 1. Baseline model & 379.60 & 401.58 & $0.680(0.618-0.741)$ \\
\hline Model 2. Baseline model+CTP based EVT eligibility (rCBF) & 379.38 & 405.03 & $0.689(0.628-0.750)$ \\
\hline Model 3. Baseline model+CTP based EVT eligibility (aCBF) & 381.57 & 407.21 & $0.680(0.618-0.741)$ \\
\hline Model 4. Baseline model+CTP based EVT eligibility (CBV) & 380.06 & 405.70 & $0.688(0.627-0.749)$ \\
\hline Model 5. Baseline model+mCTA treatment decision & 379.94 & 405.59 & $0.686(0.625-0.747)$ \\
\hline \multicolumn{4}{|l|}{$\mathrm{mRS} 0-2$ at 90 days } \\
\hline Model 1. Baseline model & 341.50 & 363.48 & $0.764(0.709-0.818)$ \\
\hline Model 2. Baseline model+CTP based EVT eligibility (rCBF) & 343.38 & 369.02 & $0.764(0.710-0.819)$ \\
\hline Model 3. Baseline model+CTP based EVT eligibility (aCBF) & 338.93 & 364.57 & $0.773(0.719-0.826)$ \\
\hline Model 4. Baseline model+CTP based EVT eligibility (CBV) & 343.34 & 368.98 & $0.763(0.709-0.817)$ \\
\hline Model 5. Baseline model+mCTA treatment decision & 341.61 & 367.25 & $0.769(0.715-0.822)$ \\
\hline
\end{tabular}

Statistical models comparing the various imaging selection criteria in estimating major clinical improvement, defined as 24-hour National Institutes of Health Stroke Scale (NIHSS) drop >50\% compared to baseline, and modified Rankin Scale 0-2 at 90 days. This analysis was only in subjects with anterior circulation large vesselocclusion $(n=289)$.

AIC, Akaike information criterion; BIC, Bayesian information criterion; Cl, confidence interval; CTP, computed tomography perfusion; EVT, endovascular treatment; rCBF, relative cerebral blood flow; aCBF, absolute cerebral blood flow; CBV, cerebral blood volume; mCTA, multi-phase computed tomography angiography.

*The baseline model incorporates onset to computed tomography time, endovascular treatment decision, baseline NIHSS, baseline Alberta Stroke Program Early CT Score (ASPECTS) and patient age. mCTA: EVT treatment decision (yes is indicated by presence of a large vessel occlusion and good or intermediate collaterals), CTP treatment decision (yes is indicated by an ischemic core volume [defined as $\mathrm{rCBF}<30 \%$ for model 2 , a cerebral blood flow $<7 \mathrm{~mL} / 100 \mathrm{~g} / \mathrm{min}$ for model 3, CBV $<2 \mathrm{~mL} / 100 \mathrm{~g}$ for model 4$]$ ) $<70 \mathrm{~mL}$ and absolute mismatch $>15 \mathrm{~mL}$ and penumbra (defined as Tmax $>6$ seconds)/core mismatch ratio $>1.8$ [DEFUSE-3] criteria). Adjustment was performed for the following pre-specified baseline variables: age, baseline stroke severity as measured by the NIHSS, time from stroke symptom onset to baseline non-contrast head computed tomography (NCCT), baseline ASPECTS (11 point ordinal scale) on NCCT, occlusion location (anterior circulation large vessel occlusion vs. not) and treatment type (EVT vs. not). Since large vessel occlusion presence was part of the mCTA and CTP-based treatment decision rules, it was not incorporated as an independent variable in models 2-5. 


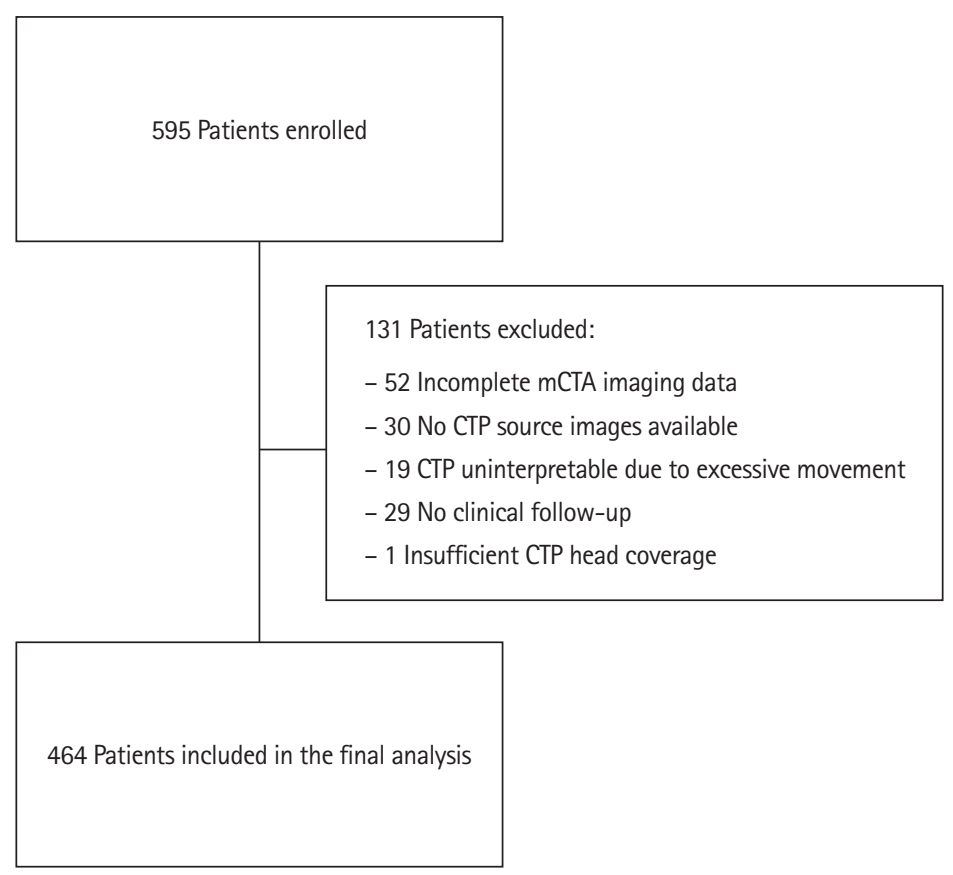

Supplementary Figure 1. Flow chart of initially enrolled and excluded patients. mCTA, multi-phase computed tomography angiography; CTP, computed tomography perfusion.

Grey circles: anterior circulation LVO

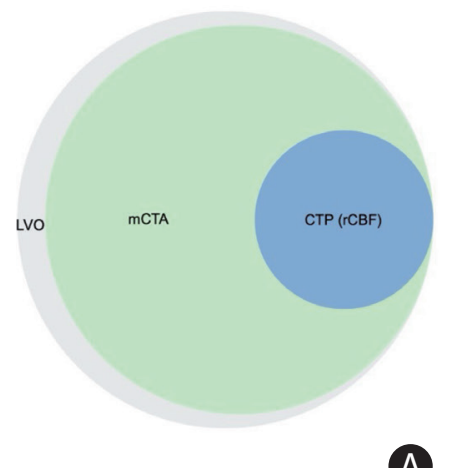

Green circles: eligible according to mCTA

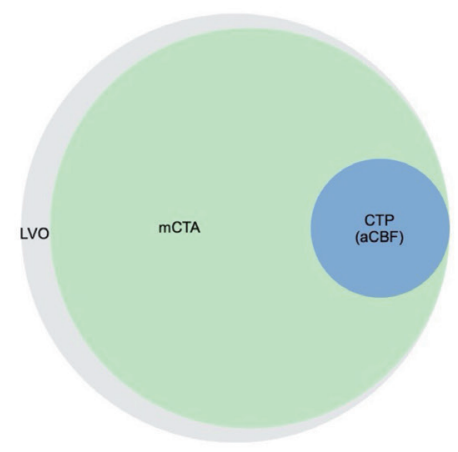

Blue circles: eligible according to CTP

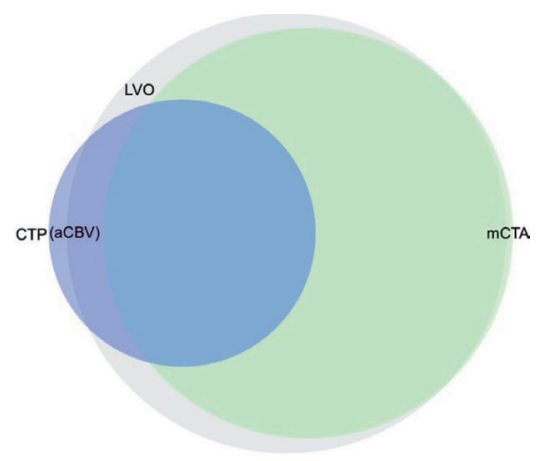

B

Supplementary Figure 2. Proportional Venn diagrams illustrating the number of patients with anterior circulation large vessel occlusion (LVO), i.e., target for endovascular treatment (EVT; grey circles), and EVT eligible patients based on computed tomography perfusion (CTP) criteria (blue circles) and multiphase computed tomography angiography $(\mathrm{mCTA})$ criteria (green circles) in patients presenting beyond 6 hours $(\mathrm{n}=69)$. (A) illustrates CTP based EVT-eligibility for relative cerebral blood flow (rCBF) $<30 \%$ as core threshold, (B) illustrates CTP based EVT-eligibility for absolute cerebral blood flow (aCBF) $<7 \mathrm{~mL} / 100$ $\mathrm{g} / \mathrm{min}$ as core threshold, and (C) illustrates CTP based EVT-eligibility for absolute cerebral blood volume $(\mathrm{aCBV})<2 \mathrm{~mL} / 100 \mathrm{~g}$ as core threshold. Note that the slight mismatch between the right margin of the green and grey circles in $(C)$ is related to the diagram type; there were no patients without LVO who are EVT eligible based on mCTA criteria. 